\title{
Michel Brix, Nerval et l'Inde des romantiques
}

\section{Annalisa Bottacin}

\section{(2) OpenEdition}

\section{Journals}

\section{Edizione digitale}

URL: http://journals.openedition.org/studifrancesi/6357

DOI: 10.4000/studifrancesi.6357

ISSN: 2427-5856

\section{Editore}

Rosenberg \& Sellier

\section{Edizione cartacea}

Data di pubblicazione: 1 novembre 2010

Paginazione: $570-571$

ISSN: 0039-2944

\section{Notizia bibliografica digitale}

Annalisa Bottacin, «Michel Brix, Nerval et I'Inde des romantiques», Studi Francesi [Online], 162 (LIV | III) | 2010, online dal 30 novembre 2015, consultato il 13 janvier 2021. URL: http://

journals.openedition.org/studifrancesi/6357 ; DOI: https://doi.org/10.4000/studifrancesi.6357

Questo documento è stato generato automaticamente il 13 janvier 2021.

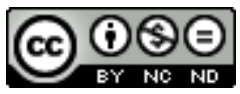

Studi Francesi è distribuita con Licenza Creative Commons Attribuzione - Non commerciale - Non opere derivate 4.0 Internazionale. 


\title{
Michel Brix, Nerval et l'Inde des romantiques
}

\author{
Annalisa Bottacin
}

\section{NOTIZIA}

MICHEL BRIX, Nerval et l'Inde des romantiques, in «Comparatio», Band 1, Heft 2, 2009, pp. 181-193.

1 L'Oriente è il viaggio verso l'arcano: il viaggio medievale è essenzialmente rivolto a Oriente. L'Oriente è la direzione per eccellenza; il senso spirituale del tutto. Andare a Oriente significa attraversare i cinque fiumi paradisiaci e avvicinarsi al paradiso terrestre. Lévi-Strauss reputa questa direzione quella del compimento: un movimento rintracciabile nel polarizzarsi delle ricchezze secondo quest'asse, nella direzione della disposizione dei quartieri, dei fenomeni di migrazione. L'India, la Persia, la Mesopotamia, l'Egitto: il viaggio verso Oriente assume un significato preciso diventando una specie di percorso à rebours, un ritorno verso ciò che è stato, una ricerca dell'origine, come scrive Schlegel, alludendo all'India, in una lettera a Tieck del 1803. Il cammino verso Oriente è un'uscita dal reale autentico, un'immersione nel rêve. È ciò che fa del viaggio in Oriente un tema ricorrente nella letteratura romantica, così in Gautier e in special modo in Nerval, che superato il Medio Oriente va alla ricerca di nuovi rituali, di modo che, come ben rileva Michel Brix in questo bel contributo, «[1]'Inde se trouva ainsi appelée à remplacer l'Égypte dans les spéculations des adeptes de l'ésotérisme et de la théosophie» (p.182). Sempre più percependo quell'insieme di teorie sopraggiunte da terre lontane, i romantici francesi sono profondamente investiti da quel misterioso mondo inconnu, che si fa sempre più spazioso nei loro scritti. Su quel «berceau du monde», come Mme de Staël definisce l'India, venivano pian piano a concretizzarsi alcuni lavori teorici preparatori per quella che Edgar Quinet definirà la «Renaissance orientale» del XIX secolo: dall'École de langues orientales vivantes, voluta da Silvestre de Saci, all'istituzione di una cattedra di sanscrito al Collège de France, alla creazione della Société Asiatique de Paris e del periodico «Journal asiatique», destinato 
a ospitare i lavori dei suoi membri. «Après l'Angleterre et l'Allemagne, - nota il critico c'est en France que se déplace progressivement le foyer des études orientales» (p. 183).

2 Profondamente attratto dai paradisi d'Oriente, Gérard de Nerval, dal 1838 feuilletoniste al «Messager de chambre», «rédige un article sur la présentation à Paris d'une troupe de bayadères» (p. 185), in cui, tra le varie considerazioni, evoca una comunione tra la razza celtica e quella indù, idea portata avanti nel tempo, in quanto nel 1841, chiederà a un funzionario del Ministero dell'Interno, Auguste Cavé, un finanziamento per effettuare un viaggio attraverso la Francia, al fine di ritrovare le prove di tale asserzione. In quegli stessi anni Quaranta, entra nell'opera nervaliana il motivo della metempsicosi, dai sonetti mistagogici delle Chimères alle Filles $d u f e u$, in quanto Gérard ritrova nelle dottrine orfiche dei Pitagorici e nell'India la religiosa credenza dell'eterna vita delle anime in perpetua trasmigrazione. Comunque è nel Voyage en Orient, pubblicato nel 1851, resoconto di un effettivo spostamento verso Oriente effettuato nel 1843 - Nerval visiterà Il Cairo, Beirut e giungerà con varie tappe fino a Costantinopoli che l'India non è mai dimenticata, è un universo sotterraneo che affiora lungo tutto il testo, e che porta alla superficie le rivelazioni dell'occulto nei percorsi del remoto e negli enigmi iniziatici, in una spasmodica Recherche de l'Absolu.

Coetaneo di Nerval, anche Théophile Gautier, codificatore della nuova teoria de l'Art pour l'Art, percepisce profondamente la fioritura di un pittoresque dai tratti strettamente ancorati a rituali, sovente danzati, e a forme orientaleggianti, che non poco influenzeranno alcune sue scelte scritturali. Per altro, nel Voyage en Italie, allorché giunge alla locanda di posta del villaggio di Simplon, non manca di attrarre l'attenzione del lettore sulla carta da parati della sala da pranzo che, «représentant la conquête des Indes par les Anglais [...] eût pu servir d'illustration à la guerre du Nizam de Méry» (Paris, Charpentier, 1884, p. 24), quello stesso Joseph Méry che «s'était fait de l'Inde une spécialité: il avait notamment rédigé un cycle "hindou" de trois romans» tra cui la Guerre du Nizam, del 1847, cui alluderà Gautier tre anni dopo nella redazione del suo periplo italiano. Comunque Méry contribuì non poco ad accrescere l'interesse di Nerval verso la letteratura indiana e in particolar modo, all'epoca, «Nerval [...] éprouvait à coup sûr un intérêt certain pour Le Chariot d'enfant, puisqu'il entreprit de le faire connaître à un très large public en France, par l'adaptation que fut reçue puis créée en 1850 au théatre de l'Odéon. Notre auteur ne s'était pas lancé seul dans une telle aventure: il s'était assuré la collaboration de Joseph Méry, qui partagea avec lui les honneurs de l'affiche» (p.187). Fu questa una pièce che caratterizzò non poco la produzione nervaliana. Il Carretto d'argilla è infatti il dramma più importante dopo la Sakuntala e data del V secolo; di soggetto buddistico è tratta dal Mritchtchkati e fu attribuita a re Soudraka. Nerval già ne era a conoscenza, in quanto ne parla in un articolo uscito su «Le Monde dramatique» nel 1835. Le Chariot d'enfant, che aveva avuto una prima traduzione dal sanscrito in inglese e quindi in lingua tedesca, solo nel 1828 ebbe un'edizione francese a cura di Alexandre Langlois; l'adattamento di Nerval e Méry $\mathrm{fu}$ accolto con grande entusiasmo dal pubblico parigino, sia alla prova generale che alla prima rappresentazione, il 13 maggio 1850, come lo stesso Méry relaziona in Les Uns et les Autres (1864), ma poco dopo la pièce fu tolta dal cartellone, per sparire definitivamente, lasciando Nerval in preda a una profonda amarezza. Poco lo consolò il fatto di aver trovato un editore per la pubblicazione.

Brix chiude quest'interessante studio, chiedendosi come la critica abbia salutato il lavoro dei due scrittori, se fosse realmente pertinente al gusto, in quanto «la pièce 
offrait une belle occasion, en effet, pour rappeler la thèse de la révélation "primordiale" et universelle» (p. 192); è comunque certo che anch'essa ebbe un ruolo fondamentale nell'ultima produzione nervaliana: un forte riflesso dell'India si percepisce ancora nell'incompiuta Aurélia. 